\title{
A Prospective Multicenter Study of Partially Covered Metal Stents in Patients Receiving Neoadjuvant Chemotherapy for Resectable and Borderline Resectable Pancreatic Cancer: BTS-NAC Study
}

\author{
Kei Saito ${ }^{1}$, Yousuke Nakai ${ }^{1}$, Hiroyuki Isayama ${ }^{1,2}$, Ryuichi Yamamoto ${ }^{3}$, Kazumichi Kawakubo ${ }^{4}$, Yuzo Kodama ${ }^{5}$, \\ Akio Katanuma ${ }^{6}$, Atsushi Kanno ${ }^{7}$, Masahiro Itonaga ${ }^{8}$, and Kazuhiko Koike ${ }^{1}$ \\ 1'Department of Gastroenterology, Graduate School of Medicine, The University of Tokyo, ${ }^{2}$ Department of Gastroenterology, Graduate \\ School of Medicine, Juntendo University, Tokyo, ${ }^{3}$ Department of Gastroenterology and Hepatology, Saitama Medical Center, Saitama \\ Medical University, Saitama, ${ }^{4}$ Department of Gastroenterology and Hepatology, Faculty of Medicine and Graduate School of Medicine, \\ Hokkaido University, Sapporo, ${ }^{5}$ Department of Gastroenterology and Hepatology, Graduate School of Medicine, Kyoto University, \\ Kyoto, ${ }^{6}$ Department of Gastroenterology, Teine-Keijinkai Hospital, Sapporo, ${ }^{7}$ Division of Gastroenterology, Tohoku University Graduate \\ School of Medicine, Sendai, and ${ }^{8}$ Second Department of Internal Medicine, Wakayama Medical University, Wakayama, Japan
}

\section{Article Info}

Received August 30, 2019

Revised January 8, 2020

Accepted January 8, 2020

Published online April 29, 2020

\section{Corresponding Author}

Hiroyuki Isayama

ORCID https://orcid.org/0000-0002-3308-9326

E-mail isayama-tky@umin.ac.jp
Background/Aims: The aim of this study was to evaluate the safety and efficacy of partially covered self-expandable metallic stents (PCSEMS) in patients undergoing neoadjuvant chemo (radio) therapy (NAC) for pancreatic cancer (PC).

Methods: This was a prospective multicenter study to evaluate the safety and efficacy of PCSEMS in patients receiving NAC for resectable and borderline resectable PC. The primary endpoint was the rate of recurrent biliary obstruction (RBO).

Results: Twenty-six patients with PC (three with resectable PC and 23 with borderline resectable PC) who underwent NAC at seven Japanese centers were included in the analysis. Both the technical and functional success rates of PCSEMS placement were $100 \%$. Early stent-related complications were observed in three patients (11.5\%): mild pancreatitis $(n=2)$ and mild liver abscess $(n=1)$. The median time to surgery or palliation was 4.0 months. Surgical resection was eventually performed in $73.1 \%$ of patients, and stent removal during surgery was successful in all patients. RBO was observed in nine patients (34.6\%): seven with stent occlusion, one with kinking and one with migration. The RBO rates in resected cases and nonresected cases were $36.8 \%$ and $28.6 \%$, respectively.

Conclusions: Biliary drainage by PCSEMS was safe and feasible in patients undergoing NAC for resectable and borderline resectable PC. (Gut Liver 2021;15:135-141)

Key Words: Pancreatic neoplasms; Neoadjuvant therapy; Self expandable metallic stents; Biliary obstruction

\section{INTRODUCTION}

Pancreatic cancer (PC), especially in the head of pancreas, is often complicated by obstructive jaundice. While endoscopic metal stent placement is the standard of care in cases with malignant biliary obstruction due to unresectable $\mathrm{PC},{ }^{1-3}$ routine preoperative biliary drainage $(\mathrm{PBD})$ is not recommended in cases undergoing upfront surgery due to the lack of advantage in PBD. ${ }^{4}$ However, early surgery is not always possible and biliary drainage is necessary in cases with concomitant cholangitis or in cases undergoing neoadjuvant chemo(radio) therapy (NAC). ${ }^{5}$

NAC is increasingly utilized in cases with borderline resectable PC (BR-PC) as well as resectable PC (R-PC) because survival after upfront surgery is still suboptimal due to the high incidence of early recurrence and the low completion rate of adjuvant chemotherapy after invasive pancreatic resection. ${ }^{6,7}$ Although self-expandable metallic stents (SEMS) appear to be superior to plastic stents (PS) in terms of stent patency in the neoadjuvant setting, there 
were no significant differences in the cost effectiveness between PS and SEMS in a small randomized controlled trial (RCT). ${ }^{8}$ Clinical outcomes of PBD might vary by the settings, i.e. stage (R-PC vs BR-PC), duration of NAC and so on, but data in Asian countries are still lacking. Since some cases without response to NAC would be convert to palliative chemotherapy, partially covered SEMS (PCSEMS), which are shown to provide better stent patency in unresectable PC, ${ }^{2}$ might be ideal in this setting. We previously reported a pilot study of PBD using fully covered metal stents for R-PC undergoing upfront surgery. ${ }^{9}$ The aim of this BTS-NAC study was to evaluate the safety and efficacy of a partially-covered metal stents bridge-to-surgery (BTS) in patients undergoing NAC for R-PC and BR-PC in Japan. The study was conducted as a pilot study for our future large-scale RCT in Japan.

\section{MATERIALS AND METHODS}

\section{Study design}

This study was approved by the Ethics Committee of the University of Tokyo (approval number: 10231). This was a single arm, multicenter prospective study. The study protocol was approved by the ethical committee at each center and written informed consent was obtained from each patient. The study was registered with the University Hospital Medical Information Network Clinical Trials Registry (UMIN000011855).

Consecutive patients with R-PC or BR-PC undergoing NAC were enrolled in seven Japanese referral centers. Inclusion criteria were the following: (1) patients with biopsy-confirmed pancreatic ductal adenocarcinoma; (2) patients with R-PC and BR-PC scheduled for neoadjuvant chemotherapy or neoadjuvant chemoradiation therapy; and (3) signs and symptoms of obstructive jaundice or acute cholangitis. Exclusion criteria were the following: (1) previous biliary drainage for 2 weeks and more; (2) prior biliary SEMS placement; (3) tumor involvement to the orifice of cystic duct; and (4) not suitable for endoscopic transpapillary biliary drainage such as severe gastric outlet obstruction.

Patients who proceeded to surgery were followed for 30 days post-surgery and patients who did not reach curative intent surgery proceeded to nonoperative, palliative chemotherapy and were followed until their deaths.

\section{National Comprehensive Cancer Network guidelines}

Patients were diagnosed with R-PC and BR-PC based on National Comprehensive Cancer Network guidelines established in $2012 .^{10}$

\section{Endoscopic procedure and stent insertion}

PCSEMS (partially covered WallFlex stent; Boston Scientific Japan, Tokyo, Japan) (Fig. 1) was used in all patients. Endoscopic retrograde cholangiography was performed under conscious sedation using pethidine and midazolam. Endoscopic sphincterotomy was performed in all cases prior to PCSEMS placement. Stent length was determined based on the stricture length on cholangiogram. The proximal end of the stent was placed at least $2 \mathrm{~cm}$ below the hepatic hilum and the distal end was placed in the duodenum.

\section{Outcome measurements}

The primary endpoint of this study was the rate of recurrent biliary obstruction (RBO) during SEMS placement until surgical resection when applicable or transition to palliative chemotherapy. The secondary endpoints were time to RBO, the causes of RBO, the rate and causes of complications other than RBO, overall survival (OS), the rate of curative surgical resection and postoperative complications.

\section{Definitions}

$\mathrm{RBO}$ and complications other than $\mathrm{RBO}$ were defined according to TOKYO criteria 2014. ${ }^{11}$ Re-intervention was defined as any type of endoscopic or percutaneous procedure necessary to improve biliary drainage for jaundice or cholangitis after placement of SEMS. Time to RBO (TRBO) was defined as time from stent insertion to RBO. Cases that underwent curative surgical resection and received palliative chemotherapy without RBO were censored. OS

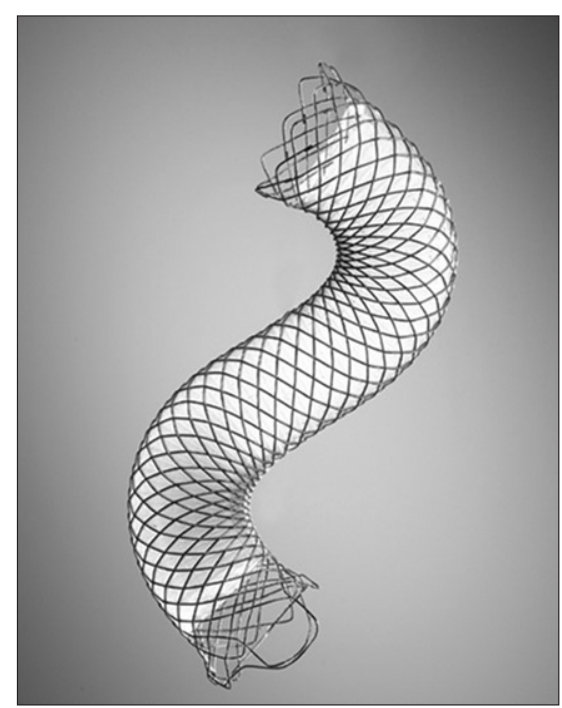

Fig. 1. A partially covered WallFlex stent. The image was used by Boston Scientific Japan, with permission. 
was defined as time from stent insertion to the final followup or death from any cause.

\section{Statistical analysis}

Formal sample size calculation was not performed because this study was a pilot feasibility study. The sample size was determined to be 30 patients during the 3-year study enrollment based on the estimated annual number of patients with $\mathrm{PC}$ receiving neoadjuvant chemotherapy and chemoradiation therapy. TRBO and OS were calculated using the Kaplan-Meier method. All statistical analysis was performed using the JMP ${ }^{\circledR} 11$ (SAS Institute Inc., Cary, NC, USA).

\section{RESULTS}

Among 30 patients who gave written informed consent for the trial between December 2013 and August 2016, four patients were excluded because of the lack of pathological confirmation $(n=3)$ and indwelling biliary drainage for more than 2 weeks $(n=1)$. As a result, 26 patients were eligible for the analysis (Fig. 2).

\section{Patient characteristics}

Patient characteristics are shown in Table 1. Median age was 68 years (range, 49 to 83 years) and the clinical stage according to the sixth edition of the International Union Against Cancer was IIa in 14, IIb in four, and III in eight. Median serum bilirubin level was $6.8 \mathrm{mg} / \mathrm{dL}$ (range, 0.4 to $23 \mathrm{mg} / \mathrm{dL}$ ) and carbohydrate antigen 19-9 value was $341.3 \mathrm{U} / \mathrm{mL}$ (range, 1 to $10,618 \mathrm{U} / \mathrm{mL}$ ). Neoadjuvant chemotherapy and chemoradiation therapy were administered in 17 patients $(65.4 \%)$ and nine patients (34.6\%), respectively. One-year and 2-year survival rates were $91.0 \%$ and $76.4 \%$, respectively.

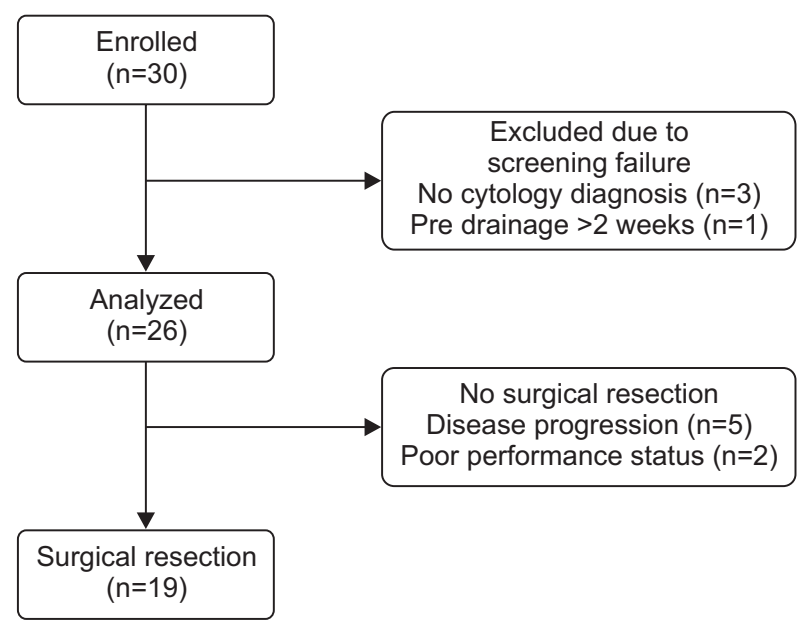

Fig. 2. Flowchart of study enrollment.

\section{Stent placement}

Stent diameter was $10 \mathrm{~mm}$ in all cases and stent length was $60 \mathrm{~mm}$ in 21 patients $(80.8 \%)$, and $80 \mathrm{~mm}$ in five patients (19.2\%). Technical and functional success rates were both $100 \%$.

\section{RBO and other complications}

The RBO was observed in $34.6 \%(9 / 26)$ and the cumulative median TRBO was not reached (Table 2). In cases that eventually underwent surgical resection after NAC, the rate of RBO was $36.8 \%$ (7/19) with the median TRBO of 142 days. Meanwhile, the RBO rate in patients who did not undergo surgical resection after NAC was $28.6 \%(2 / 7)$ and the median TRBO was not reached. The rate of RBO

Table 1. Patient Characteristics

\begin{tabular}{lc}
\hline \multicolumn{1}{c}{ Variable } & Value \\
\hline Age, yr & 68 (49-83) \\
Male sex & 14 (53.8) \\
Stage (UICC), Ila/IIb/III & $14 / 4 / 8$ \\
Neoadjuvant therapy & $17(65.4)$ \\
Chemotherapy & $9(34.6)$ \\
Chemoradiation therapy & \\
Chemotherapy regimen & $5(19.2)$ \\
Gemcitabine plus nab-paclitaxel & $1(3.8)$ \\
FOLFIRINOX & $3(11.5)$ \\
Gemcitabine plus S-1 & $5(19.2)$ \\
Gemcitabine & $6(23.1)$ \\
S-1 & $6(23.1)$ \\
Others & $6.8(0.4-23.0)$ \\
Initial total bilirubin, mg/dL & $341.3(1-10,618)$ \\
Preoperative CA 19-9, U/mL & $4.0(2.1-33.8)$ \\
Preoperative biliary drainage period, mo &
\end{tabular}

Data are presented as median (range) or number (\%).

UICC, International Union Against Cancer; FOLFIRINOX, irinotecan, oxaliplatin, leucovorin, and fluorouracil (5-FU); CA 19-9, carbohydrate antigen 19-9.

Table 2. Recurrent Biliary Obstruction and Complications

\begin{tabular}{lccc}
\hline \multicolumn{1}{c}{ Variable } & Incidence & $\begin{array}{c}\text { Time to } \\
\text { RBO, day }\end{array}$ & $\begin{array}{c}\text { Time to com- } \\
\text { plications, day }\end{array}$ \\
\hline RBO & & & \\
Overall & $9(34.6)$ & & \\
Occlusion & $7(27.0)$ & $53(2-142)$ & \\
Non-occlusion cholangitis & 0 & & \\
Kinking & $1(3.8)$ & 4 & 1 and 2 \\
Migration & $1(3.8)$ & 60 & \\
Complications other than RBO & & & \\
Pancreatitis & $2(7.7)$ & & 21 \\
Cholecystitis & 0 & & \\
Bleeding & 0 & & \\
Perforation & 0 & & \\
Liver abscess & $1(3.8)$ & & \\
\hline
\end{tabular}

Data are presented as number (\%) or median (range). RBO, recurrent biliary obstruction. 


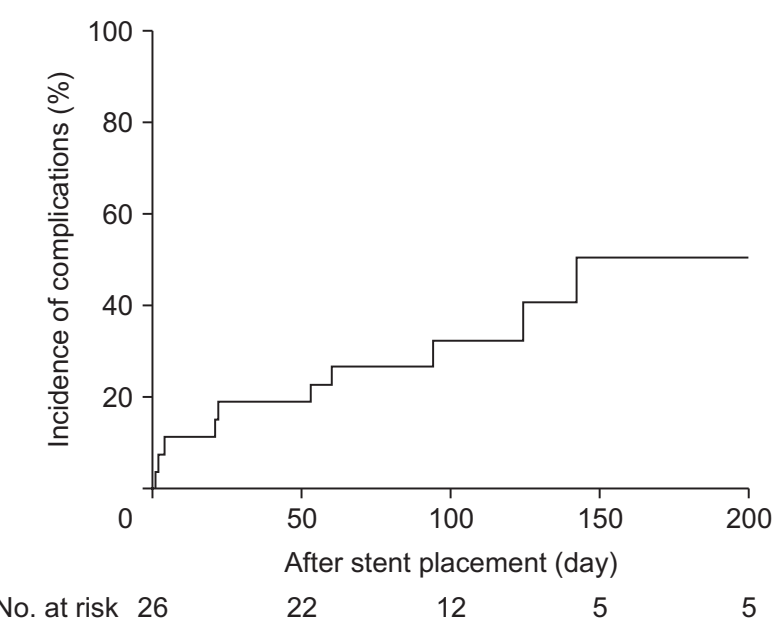

No. at risk 26

22

12

Fig. 3. Cumulative incidence of stent-related recurrent biliary obstruction and adverse events in 26 patients who underwent partially covered metal stent placement.

in patients undergoing neoadjuvant chemoradiation and chemotherapy alone were $75.0 \%$ and $16.7 \%$, respectively $(\mathrm{p}=0.004)$. The causes of RBO were seven stent occlusions due to biliary sludge, one migration and one kinking, and tumor ingrowth was not observed. Stent exchange was performed in six and balloon sweep was performed in three as re-interventions; no percutaneous intervention was not performed.

Stent-related complications other than RBO were observed in three patients (Table 2). Two patients (7.7\%) developed mild post-endoscopic retrograde cholangiopancreatography pancreatitis after stent placement, which resolved without removal of SEMS. One patient (3.8\%) developed liver abscess 21 days after SEMS placement. Liver abscess was successfully managed by antibiotics alone without any interventions.

A cumulative composite incidence of RBO and other stent-related complications is shown in Fig. 3 with the cumulative RBO incidence of $32.5 \%$ at 100 days and $50.8 \%$ at 200 days.

\section{Neoadjuvant therapy and curative surgical resection}

Tumor response by RECIST criteria ${ }^{12}$ was partial response in three and stable disease in 20 , and three cases were not evaluable for tumor response. No radiological complete response was observed. As a result, the response rate was $11.5 \%$ and the disease control rate was $88.5 \%$.

During the clinical course of NAC, seven patients were diagnosed as unresectable and proceeded to palliative treatment: five due to disease progression and two due to deteriorated performance status. The median time to palliation was 385 days.

The remaining 19 patients $(73.1 \%)$ proceeded to $\mathrm{cu}-$ rative surgical resection with a median time to surgery of 105 days. Median operative blood loss was $1,017 \mathrm{~mL}$ (range, 100 to $4,400 \mathrm{~mL}$ ) and median operative time was 555 minutes (256 to 897 minutes). There was no postoperative mortality, but three postoperative morbidities were observed in two patients (10.5\%): two bile leakage and one intra-abdominal abscess. No technical difficulties were reported in stent extraction or creation of hepaticojejunostomy during surgical resection.

\section{DISCUSSION}

In this multicenter prospective study, PBD using PCSEMS was feasible and safe with the technical success rate of $100 \%$ and the complication rate of $11.5 \%$ in cases with $\mathrm{R}-\mathrm{PC}$ and BR-PC undergoing NAC. The RBO rate was $34.6 \%$ with the median time to surgery or palliation of 4.0 months.

In patients undergoing $\mathrm{NAC}$ for $\mathrm{PC}, \mathrm{RBO}$ can cause delay or discontinuation of NAC and affect treatment outcomes. The reported rates of $\mathrm{RBO}$ were $15 \%$ to $35 \%$ when SEMS was used as $\mathrm{PBD}{ }^{8,13,14}$ The $\mathrm{RBO}$ rate in our study was $34.6 \%$, which was relatively high. There is no consensus on the indication, regimen, and duration of NAC for PC. Therefore, the resection rate and time to surgery after NAC depend on the tumor status and each institution's protocol. If patients with potentially R-PC are enrolled, then, the duration of NAC can be short with a high surgical resection rate. Meanwhile, if patients with locally advanced PC are included, the duration of NAC can be long and conversion rate to palliation will be high, which can affect the rate of RBO and TRBO. For example, Pop et al. ${ }^{15}$ reported the complication rate including $\mathrm{RBO}$ was $14.8 \%$ but the median time to surgery was only 32 days. Meanwhile, in our study, time to surgery was relatively long (105 days at median). The addition of radiation might also affect outcomes of PBD during NAC; the rates of RBO were $75.0 \%$ and $16.7 \%$ in patients undergoing chemoradiation therapy and chemotherapy alone, though the number of cases was small. Two possible reasons for the high RBO rate in chemoradiation therapy are considered. First, the duration and severity of neutropenia might increase by adding radiation therapy to chemotherapy. Second, radiation can cause some inflammation and edema in the duodenum. We previously reported that RBO would increase in cases with duodenal invasion. ${ }^{16}$ It is possible that radiation would increase duodenobiliary reflux and subsequent cholangitis and RBO. Kubota et al. ${ }^{14}$ reported that the median TRBO was longer in PCSEMS (97 days vs 55 days in PCSEMS and PS groups) but that the re-intervention rate 
was still as high as $23.5 \%$ in PCSEMS during the 3-month period of NAC. Since there are no consensus on the indication (R-PC and/or BR-PC), regimen and duration of NAC, inter-study comparison is difficult and only a prospective RCT can clarify appropriate stent selection during NAC.

While SEMS appears to provide better stent patency than PS both in resectable and unresectable malignant biliary obstruction, there are still some concerns on the use of SEMS as PBD because of possible inflammation along the bile duct. However, the use of SEMS was reportedly not associated with overall postoperative complications, hospital stay or mortality ${ }^{5,17}$ despite a higher wound infection rate and longer operation time. In our study, there were no postoperative mortality and three morbidities were observed in two patients (10.5\%), which suggested that PCSEMS did not adversely affect surgical outcomes. In general, the use of PBD is associated with postoperative infectious complications and in a previous study the rate of wound infection was significantly higher in SEMS: $31.0 \%$ in SEMS, $12.8 \%$ in PS and $6.2 \%$ in non-stented group $(\mathrm{p}<0.001){ }^{5}$ The effects of PBD on postoperative complications are clinically important since the introduction of adjuvant chemotherapy is essential even after $\mathrm{R} 0$ resection of PC. ${ }^{18}$ Thus, the effects of the stent type on postoperative infections, the introduction of adjuvant chemotherapy and OS, should be further evaluated.

Although a recent Japanese RCT demonstrated that PCSEMS showed better stent patency than uncovered SEMS in unresectable distal malignant biliary obstruction, ${ }^{2}$ conflicting data have been reported on the comparison of covered and uncovered SEMS. ${ }^{19,20}$ While covered SEMS prevents tumor ingrowth, it is associated with stent migration. Few data are available on covered versus uncovered SEMS in the neoadjuvant setting. Only one international RCT showed non-inferiority of PCSEMS to uncovered SEMS in cases undergoing NAC for PC but further investigations are necessary. ${ }^{21}$

In unresectable malignant biliary obstruction, we previously reported that fully covered SEMS (FCSEMS) and PCSEMS were comparable in safety and effectiveness including stent migration (14\% in FCSEMS and $8 \%$ in PCSEMS). ${ }^{22,23}$ While FCSEMS allows easy stent removal at surgical resection, PCSEMS theoretically decreases the chance of stent migration even after tumor shrinkage by NAC, especially when a longer ( $>6$ months) duration of NAC is expected. ${ }^{23}$ It is still unclear whether either FCSEMS or PCSEMS is preferable as PBD in the neoadjuvant setting.

Post-endoscopic retrograde cholangiopancreatography pancreatitis was observed in $7.7 \%$ in our study. Although pancreatitis was mild in our study, severe pancreatitis can delay the introduction of NAC as well as potentially in- terfere with surgical procedures due to the peripancreatic inflammation. SEMS with high axial force and non-PC were independent risk factors for pancreatitis after SEMS placement. ${ }^{24}$ There have been no data supporting routine endoscopic sphincterotomy prior to metal stent placement for prevention of pancreatitis in unresectable malignant biliary obstruction. ${ }^{25,26}$ An earlier stage of PC might be associated with a relatively higher incidence of pancreatitis because pancreatic duct obstruction with upstream pancreatic atrophy is more often seen in advanced PC and the necessity of endoscopic sphincterotomy prior to $\mathrm{PBD}$ should further be clarified in the early stage of PC. We excluded cases with tumor involvement to orifice of the cystic duct, a risk factor for cholecystitis, ${ }^{27}$ and no cholecystitis was observed in our study. Although we excluded those with tumor involvement to orifice of the cystic duct in our previous study of SEMS as PBD without NAC, the rate of cholecystitis was $8 \% .{ }^{9}$ Previous studies showed no significant differences in the rate of cholecystitis between uncovered and covered metal stents for unresectable and BR malignant biliary obstruction. ${ }^{27,28}$ Since cholecystitis can delay surgery or NAC, it is further to be explored how we can reduce the risk of cholecystitis.

Our study has some limitations including the small sample size and the lack of long-term outcomes after surgical resection. In addition, clinical outcomes of PBD might differ depending on the time to surgery and an RCT is warranted to confirm the superiority of covered SEMS to PS in the neoadjuvant setting of both R-PC and BR-PC.

In conclusion, the use of PCSEMS was feasible and safe as $\mathrm{PBD}$ in the setting of NAC for PC. We are planning a large-scale multicenter RCT of PCSEMS and PS as PBD both for R-PC and BR-PC in Japan.

\section{CONFLICTS OF INTEREST}

H.I. and Y.N. have received grant support from Boston Scientific Japan. The other authors of the manuscript declare that there are no conflicts of interest.

\section{ACKNOWLEDGEMENTS}

The authors would like to thank Drs. Kei Yane (TeineKeijinkai Hospital, Sapporo, Japan), Motoyuki Tsuda (Kyoto University, Kyoto, Japan), Kazunaga Ishigaki (The University of Tokyo, Tokyo, Japan), Saburo Matsubara (Saitama Medical University, Saitama, Japan), Hiroshi Kawakami (University of Miyazaki Hospital, Miyazaki, Japan) for their patient management. 


\section{AUTHOR CONTRIBUTIONS}

Study concept and design: K.S., Y.N., H.I. Acquisition of data analysis and interpretation of data: K.S., R.Y., K.K., Y.K., A. Katanuma, A. Kanno, M.I. Drafting of the manuscript: K.S., Y.N., H.I. Critical revision of the manuscript for important intellectual content: K.S., Y.N., H.I., K.S., R.Y., K.K., Y.K., A. Katanuma, A. Kanno, M.I. Supervision: K.K.

\section{ORCID}

Kei Saito https://orcid.org/0000-0003-2290-9373

Yousuke Nakai https://orcid.org/0000-0001-7411-1385

Hiroyuki Isayama https://orcid.org/0000-0002-3308-9326

Ryuichi Yamamoto

Kazumichi Kawakubo

https://orcid.org/0000-0003-0299-5470

https://orcid.org/0000-0002-8979-9474

Yuzo Kodama https://orcid.org/0000-0002-1741-5638

Akio Katanuma https://orcid.org/0000-0003-3548-5215

Atsushi Kanno https://orcid.org/0000-0001-6341-5199

Masahiro Itonaga https://orcid.org/0000-0002-0343-7246

Kazuhiko Koike https://orcid.org/0000-0002-9787-1907

\section{REFERENCES}

1. Isayama $\mathrm{H}$, Komatsu $\mathrm{Y}$, Tsujino $\mathrm{T}$, et al. A prospective randomised study of "covered" versus "uncovered" diamond stents for the management of distal malignant biliary obstruction. Gut 2004;53:729-734.

2. Kitano M, Yamashita Y, Tanaka K, et al. Covered selfexpandable metal stents with an anti-migration system improve patency duration without increased complications compared with uncovered stents for distal biliary obstruction caused by pancreatic carcinoma: a randomized multicenter trial. Am J Gastroenterol 2013;108:1713-1722.

3. Lee JH, Krishna SG, Singh A, et al. Comparison of the utility of covered metal stents versus uncovered metal stents in the management of malignant biliary strictures in 749 patients. Gastrointest Endosc 2013;78:312-324.

4. van der Gaag NA, Rauws EA, van Eijck CH, et al. Preoperative biliary drainage for cancer of the head of the pancreas. N Engl J Med 2010;362:129-137.

5. Cavell LK, Allen PJ, Vinoya C, et al. Biliary self-expandable metal stents do not adversely affect pancreaticoduodenectomy. Am J Gastroenterol 2013;108:1168-1173.

6. Blazer M, Wu C, Goldberg RM, et al. Neoadjuvant modified (m) FOLFIRINOX for locally advanced unresectable (LAPC) and borderline resectable (BRPC) adenocarcinoma of the pancreas. Ann Surg Oncol 2015;22:1153-1159.

7. Murakami Y, Uemura K, Sudo T, et al. Survival impact of neoadjuvant gemcitabine plus S-1 chemotherapy for patients with borderline resectable pancreatic carcinoma with arterial contact. Cancer Chemother Pharmacol 2017;79:37-47.

8. Gardner TB, Spangler CC, Byanova KL, et al. Cost-effectiveness and clinical efficacy of biliary stents in patients undergoing neoadjuvant therapy for pancreatic adenocarcinoma in a randomized controlled trial. Gastrointest Endosc 2016;84:460-466.

9. Togawa $\mathrm{O}$, Isayama $\mathrm{H}$, Kawakami $\mathrm{H}$, et al. Preoperative biliary drainage using a fully covered self-expandable metallic stent for pancreatic head cancer: a prospective feasibility study. Saudi J Gastroenterol 2018;24:151-156.

10. Tempero MA, Arnoletti JP, Behrman SW, et al. Pancreatic Adenocarcinoma, version 2.2012: featured updates to the NCCN Guidelines. J Natl Compr Canc Netw 2012;10:703713.

11. Isayama H, Hamada T, Yasuda I, et al. TOKYO criteria 2014 for transpapillary biliary stenting. Dig Endosc 2015;27:259264.

12. Eisenhauer EA, Therasse P, Bogaerts J, et al. New response evaluation criteria in solid tumours: revised RECIST guideline (version 1.1). Eur J Cancer 2009;45:228-247.

13. Aadam AA, Evans DB, Khan A, Oh Y, Dua K. Efficacy and safety of self-expandable metal stents for biliary decompression in patients receiving neoadjuvant therapy for pancreatic cancer: a prospective study. Gastrointest Endosc 2012;76:6775 .

14. Kubota K, Sato T, Watanabe S, et al. Covered self-expandable metal stent deployment promises safe neoadjuvant chemoradiation therapy in patients with borderline resectable pancreatic head cancer. Dig Endosc 2014;26:77-86.

15. Pop GH, Richter JA, Sauer B, et al. Bridge to surgery using partially covered self-expandable metal stents (PCMS) in malignant biliary stricture: an acceptable paradigm? Surg Endosc 2011;25:613-618.

16. Hamada T, Isayama $H$, Nakai $Y$, et al. Duodenal invasion is a risk factor for the early dysfunction of biliary metal stents in unresectable pancreatic cancer. Gastrointest Endosc 2011;74: 548-555.

17. Singal AK, Ross WA, Guturu P, et al. Self-expanding metal stents for biliary drainage in patients with resectable pancreatic cancer: single-center experience with 79 cases. Dig Dis Sci 2011;56:3678-3684.

18. De Pastena M, Marchegiani G, Paiella S, et al. Impact of preoperative biliary drainage on postoperative outcome after pancreaticoduodenectomy: an analysis of 1500 consecutive cases. Dig Endosc 2018;30:777-784.

19. Saleem A, Leggett CL, Murad MH, Baron TH. Meta-analysis of randomized trials comparing the patency of covered and 
uncovered self-expandable metal stents for palliation of distal malignant bile duct obstruction. Gastrointest Endosc 2011;74:321-327.

20. Almadi MA, Barkun AN, Martel M. No benefit of covered vs uncovered self-expandable metal stents in patients with malignant distal biliary obstruction: a meta-analysis. Clin Gastroenterol Hepatol 2013;11:27-37.

21. Seo DW, Sherman S, Dua KS, et al. Covered and uncovered biliary metal stents provide similar relief of biliary obstruction during neoadjuvant therapy in pancreatic cancer: a randomized trial. Gastrointest Endosc 2019;90:602-612.

22. Isayama $H$, Mukai $T$, Itoi $T$, et al. Comparison of partially covered nitinol stents with partially covered stainless stents as a historical control in a multicenter study of distal malignant biliary obstruction: the WATCH study. Gastrointest Endosc 2012;76:84-92.

23. Kogure H, Ryozawa S, Maetani I, et al. A prospective multicenter study of a fully covered metal stent in patients with distal malignant biliary obstruction: WATCH-2 study. Dig
Dis Sci 2018;63:2466-2473.

24. Kawakubo K, Isayama H, Nakai Y, et al. Risk factors for pancreatitis following transpapillary self-expandable metal stent placement. Surg Endosc 2012;26:771-776.

25. CotéGA, Kumar N, Ansstas M, et al. Risk of post-ERCP pancreatitis with placement of self-expandable metallic stents. Gastrointest Endosc 2010;72:748-754.

26. Hayashi T, Kawakami H, Osanai M, et al. No benefit of endoscopic sphincterotomy before biliary placement of selfexpandable metal stents for unresectable pancreatic cancer. Clin Gastroenterol Hepatol 2015;13:1151-1158.

27. Isayama $H$, Kawabe T, Nakai $Y$, et al. Cholecystitis after metallic stent placement in patients with malignant distal biliary obstruction. Clin Gastroenterol Hepatol 2006;4:11481153.

28. Suk KT, Kim HS, Kim JW, et al. Risk factors for cholecystitis after metal stent placement in malignant biliary obstruction. Gastrointest Endosc 2006;64:522-529. 